\title{
DÉCLANCHEMENT SIMULTANÉ DE L'ESTRUS DANS UN LOT DE BREBIS AVEC POSSIBILITÉ DE GESTATION ULTÉRIEURE \\ PAR
}

\section{DA UZIER et Suzanne WINTENBERGER}

Station de Physiologie Animale, Centre National de Recherches Zootechniques, Jouy-en-Josas.

Il peut être intéressant pour les éleveurs d'obtenir l'agnelage à une époque favorable à la vente des agneaux, ou de grouper la mise-bas (production du lait, utilisation de l'insémination artificielle). Seul le premier problème a fait l'objet de nombreux travaux dont les résultats sont variables, et même contradictoires. La plupart des auteurs (COLE et MILLER, I935; Litovtchenko, I936; Pacetchnik, I936; Hammond et Parkes, I942; Bell, Casida, Bohsted' T, Darlow, I94I ; Cole, Hart, Miller, I945 ; Hammond Jr., I945 ; Zavadovski, I945 ; Thibault, Laplaud, Ortavant, I948 ; Robinson, I950) ont utilisé les hormones gonadotropes (sérum de jument gravide, le plus souvent) injectées par voie sous-cutanée en une ou deux injections espacées de 17 jours. Cette méthode ne provoque les chaleurs que chez une faible proportion de Brebis, l'ovulation se produit généralement, mais elle est rarement suivie de fécondation. En traitant 7 Brebis avec I ooo U. I. de sérum de jument gravide, 48 heures après injection sous-cutanée de $35 \mathrm{mgr}$ de progestérone RoBinson (I952) n'a obtenu que deux Brebis fécondées. De plus, faute de lot témoin, il n'était pas possible de vérifier si elles étaient réellement en anœstrus.

Thibault, Ortavant, Desbois, Laurans, I948, ont essayé en injectant des hormones gonadotropes, de la folliculine et de la progestérone, de reconstituer un cycle hormonal artificiel, mais leurs résultats ne sont pas significatifs du fait de l'entrée en rut dans le lot témoin d'un certain nombre de Brebis.

Il était donc nécessaire de reprendre ces travaux pour trouver une méthode permettant d'obtenir les chaleurs, l'ovulation et la fécondation.

Dans ce but nous avons utilisé 24 Brebis Berrichonnes $\times$ Ile-de-France, dont le rut était détecté quotidiennement, 6 lots furent constitués. Le lot I a permis de connaitre l'état des ovaires et du tractus chez les Brebis non traitées. Le $2^{\text {eme }}$ lot a servi également de lot témoin, afin de déterminer la date normale d'entrée en rut des Brebis. Les lots : 3, 4, 5, 6 ont été soumis comme l'indique le tableau I, à des injections d'hormone gonadotrope (1),

(1) Hormone gonadotrope Roussel.

Annales de Zootechnie. - I952 
et de progestérone $\left(^{1}\right)$; cette hormone injectée quotidiennement, et pendant I4 jours, bloque le cycle des Brebis en période d'activité sexuelle, et le rut survient dans les 2 à 4 jours après la fin du traitement (DUTT et CASIDA, I948; O'Mary, Pope, Casida, I950).

Tous les animaux ont été abattus entre le 9 et le $\mathbf{r} 6$ juillet.

TABLEAU I.

\begin{tabular}{|c|c|c|c|}
\hline Lot & H. Gonadotrope & Progestérone & H. Gonadotrope \\
\hline 3 & & $\begin{array}{c}\text { Io mg de progestérone par jour } \\
\text { en injection ss-cutanée du } 23^{-6} \\
\text { au } 6-7-195^{2} \text { inclus }\end{array}$ & \\
\hline 4 & $\begin{array}{l}800 \text { U. I. d'hormone gonado- } \\
\text { trope en ss-cutanée, le } 23^{-6}\end{array}$ & $\begin{array}{c}\text { Io mg de progestérone par jour } \\
\text { en injection ss-cutanée du } 26-6 \\
\text { au } 9-7^{-195^{2}} \text { inclus }\end{array}$ & \\
\hline 5 & $\begin{array}{l}800 \text { U. I. d'hormone gonado- } \\
\text { trope en ss-cutance, le } 21-6-5^{2}\end{array}$ & $\begin{array}{c}\text { Io mg de progestérone par jour } \\
\text { en injection ss-cutanée du } 24-6 \\
\text { au } 7-7-195^{2} \text { inclus }\end{array}$ & $\begin{array}{l}800 \text { U. I. d'hormone gonado- } \\
\text { trope en ss-cutanée le 7-7-52 }\end{array}$ \\
\hline 6 & & $\begin{array}{c}\text { Io mg de progestérone par jour } \\
\text { en injection ss-cutanée du } 2 \text { I-6 } \\
\text { au } 4-7-195^{2} \text { inclus }\end{array}$ & $\begin{array}{l}800 \text { U. I. d'hormone gonado- } \\
\text { trope ss-cutanée le 4-7-1852}\end{array}$ \\
\hline
\end{tabular}

\section{RESULTATS}

Toutes les Brebis du lot I avaient un tractus différencié et des ovaires fonctionnels, avec des corps jaunes provenant d'ovulations récentes (de 24 heures à IO-I2 jours) ou des follicules différenciés. Ces Brebis avaient donc des cycles non apparents sans manifestation de chaleurs.

Les premières chaleurs des Brebis du lot 2 ne se sont manifestées qu'à des intervalles compris entre $I 5$ jours et 2 mois après la fin de l'expérience (respectivement le I/8 et le Io/8, le I/9 et le I5/9/I952).

Tableau II

\begin{tabular}{|c|c|c|c|}
\hline Lot & $\begin{array}{l}\text { Brebis entrces en rut après } \\
\text { la fin du traitement }\end{array}$ & Brebis ayant ovulé & $\begin{array}{c}\text { Brebis fécondées (saillie } \\
\text { normale ou forcée) }\end{array}$ \\
\hline 3 & $2(50 \%)$ & $4(100 \%)$ & I $(25 \%)$ \\
\hline 4 & $3(75 \%)$ & $4(100 \%)$ & $2(50 \%)$ \\
\hline 5 & o & $4(100 \%)$ & $\circ$ \\
\hline 6 & $2(50 \%)$ & $3(75 \%)$ & I $(25 \%)$ \\
\hline
\end{tabular}

(1) Zooprogestryl Renault. 
Le tableau II montre que les divers traitements essayés ont provoqué dans presque tous les cas une ovulation groupée sur 3 ou 4 jours (I5 brebis sur I6). Toutefois le rut n'est apparu, et la fécondation n'a été possible, chez une proportion satisfaisante de Brebis, qu'avec une injection d'hormone gonadotrope préalable au traitement à la progestérone (lot $\left.n^{0} 4\right)$.

De l'examen des ovaires sur les animaux abattus, il ressort que, dans les lots 4 et 5 , seules 2 brebis sur 8 ont ovulé à la suite du traitement préalable à 1 'hormone gonadotrope, aucun ne présentant de rut, alors qu'après les injections de progestérone (lots 3, 4 et 5), l'ovulation s'est régulièrement produite.

On ne peut manquer de rapprocher ce résultat de la pratique utilisée dans certains cas chez les Bovins pour provoquer l'ovulation et l'œstrus après l'extirpation des corps jaunes.

D'autre part COLE, HART et MILLER (1945) ont observé que chez la Brebis, les corps jaunes formés après une première injection d'hormone gonadotrope ne présentaient pas un développement normal ; pour eux l'absence de chaleurs, après une deuxième injection d'hormone gonadotrope, pourrait résulter d'une sécrétion insuffisante de progestérone par ces corps jaunes, pendant la période s'étendant entre les deux injections d'hormone gonadotrope. De même Robinson (1952) estime qu'un pré-traitement à la progestérone, avant l'injection d'hormone gonadotrope, est nécessaire pour induire l'œstrus.

En fait une telle expérience (lot 6) ne nous a pas permis de confirmer l'opinion de ce dernier auteur, puisque les plus mauvais résultats ont été enregistrés dans ce cas. Cependant, il est possible que notre échec tienne à d'autres causes (doses d'hormone, délai ou traitements inadéquats), car nous avons effectivement remarqué que la progestérone facilite la différenciation normale du tractus et contribue à favoriser les modifications qui accompagnent une ovulation naturelle et doivent permettre la fécondation.

En résumé, ces expériences montrent que:

$I^{0}$ pendant 1'anœstrus on peut déclencher l'ovulation et les chaleurs, avec possibilité de fécondation, chez la moitié environ des Brebis traitées ; $2^{\circ}$ les meilleurs résultats ont été obtenus en injectant $800 \mathrm{U}$. I. d'hormone gonadotrope, préalablement à un traitement progestatif ;

$3^{\circ}$ la progestérone facilite la différenciation du tractus génital au repos pendant l'anœstrus.

De nouvelles recherches seront entreprises ultérieurement pour préciser et améliorer les résultats obtenus, conditions nécessaires pour la mise en application pratique de ce traitement. 


\section{BIBLIOGRAPHIE}

Bell (T. D.), Casida (L. E.), Bohsteit (G.), Darlow (A. E.). - Production of heat and ovulation in the anestrous ewe. J. Agric. Res., I94 I, 62, Iо, 619-25.

Cole (II. H.). - - On the biological properties of mare gonadotropic hormone. Amer. J. Anat., I $936,59,299-33$.

Cole (H. H.), Hart (G. H.), Miller (F. F.). - Studies on the hormonal control of estrous phenomena in the anestrous ewe. Endocrinology, I945, 36, 370-80.

Cole (H. H.), Miller (R. F.). - Changes in the reproductive organs of the ewe with some data bearing on their control. Amer. J. Anat, 1935, 57, $40-98$.

DUTT (R. H.), CASIDA (L. E.). - Alteration of the estrual cycle in sheep by use of progesterone and its effect upon subsequent ovulation and fertility. Endocrinology, 1948 43, 208-1 7 .

GreEN (W. W.), Winters (L. M.). - Study on the physiology of reproduction in the sheep. III The time of ovulation and rate of sperm travel. Anat. Rec., 1935, 61, 457-70.

HAMmond J. jr). - Induced ovulation and heat in anestrous sheep. J. Endocrinol., 1945, 4, $169-80$.

IIAMMOND (J.) Jr., Hammond (J.), Parkes (A. S.). - Ilormonal augmentation of fertility in sheep. I. Induction of ovulation, superovulation and heat in sheep: J. Agric. Sci. $1942,3^{2}, 3,308-23$.

Mc Kenzie (F.), 'Terril (C. B.). - Estrus, ovulation and related phenomena in the ewe. Res. Bull., 1937, no 264. Missouri.

Litovtchenko (I. R.). - L'action du sérum de jument gravide sur les fonctions sexuelles du mouton. Problemy Jyvolnovodstva, i936, ${ }^{\circ}$ ro.

loginova. - D'après Hammond Jr. Hammond et Parkes (1939).

O'MARY (C. C.), Pope (A. L.), CASida (L. E.). - - The use of progesterone in the synchronisation of the estrual period in a group of ewes and the effect on their subsequent lambing record. J. Animal Sci., 1950, 9, 499-503.

Pacetchnik (L. N.). - D'après Zavadovski.

ROBINSON (T. J.). - The control of fertility in sheep. Part I hormonal therapy with induction of pregnancy with anestrous ewe. J. Agric. Sci., 1950, 40, 275-307.

RoBinson (T. J.). - Rôle of progesterone in the mating behavior of the ewe. Nature, I950, $170,373-4$.

Thibault (C.), Laplaud (M.), Ortavant (R.). -- Possibilité d'une gestation supplémentaire chez les Brebis. C. R. Acad. Agr., 1948.

Thibault (C.), Ortavant (R.), I)esbois (Y.), Lacrans (R.). -- Recherches pour avancer la saison sexuelle de la Brebis et réduire à quelques jours les périodes d'insémination et d'agnelage. C. R. Acad. Agr., 1948. 